\title{
Divide and shape: an endosymbiont in action
}

\author{
Kevin A. Pyke
}

Received: 27 June 2012/ Accepted: 3 August 2012/Published online: 22 August 2012

(C) Springer-Verlag 2012

\begin{abstract}
The endosymbiotic evolution of the plastid within the host cell required development of a mechanism for efficient division of the plastid. Whilst a model for the mechanism of chloroplast division has been constructed, little is known of how other types of plastids divide, especially the proplastid, the progenitor of all plastid types in the cell. It has become clear that plastid shape is highly heterogeneous and dynamic, especially stromules. This article considers how such variation in morphology might be controlled and how such plastids might divide efficiently.
\end{abstract}

Keywords Amyloplasts · Chloroplast division · Plastid division · Plastid morphology · Proplastids · Stromules

\section{Introduction}

The evolution of the original endosymbiont, that gave rise to modern day plastids, has been a highly complex process, leading to a myriad of interactions between the evolving plastid and its host cell. Such processes have been two directional in that the plastid has evolved significantly to interact with the host cell's biochemistry and molecular systems whereas the host cell has taken on board much of the original endosymbiont genome and incorporated it into its own. Thus in the cellular interaction of the modern day

A contribution to the Special Issue on Evolution and Biogenesis of Chloroplasts and Mitochondria.

K. A. Pyke $(\bowtie)$

Plant and Crop Sciences Division, School of Biosciences,

University of Nottingham, Sutton Bonington Campus,

Loughborough LE12 5RD, UK

e-mail: kevin.pyke@nottingham.ac.uk plastid, there are many processes whereby the plastid interacts with other cellular compartments at the molecular as well as the physical level.

Paramount in this evolutionary process was the development of a system, that enabled the division of plastids within the cell in a coordinated manner. This system also interacts spatially and temporally with the operations of the cell itself and, not least, in enabling plastid division to be coordinated in relation to cell division such that each daughter cell contains a stable segregated population of plastids. Such a division process was fundamental to the biogenesis of higher and lower plants since the presence of plastids in higher plant cells is generally considered to be an absolute requirement for their normal cellular function. In the last twenty years of plastid biology research, very significant progress has been made in understanding aspects of the plastid division process, primarily that of the chloroplast, from the identification of the first Arabidopsis mutants that perturb the plastid division process (Pyke and Leech 1992) to the identification of the first prokaryotic genes in higher plants that function in chloroplast division; FtsZ (Osteryoung and Vierling 1995). Several recent reviews (Maple and Moller 2010; Miyagishima and Kabeya 2010; Pyke 2010; Miyagishima 2011; Miyagishima et al. 2011) have described how the various molecules identified as functioning in some part of the chloroplast division process have been incorporated into a model of a proposed chloroplast division mechanism. I will briefly describe something of the current model of chloroplast division, before considering other aspects of plastid division and plastid morphology, but readers are directed to these extensive reviews for an in depth description of the current thinking on the topic.

Central to the proposed mechanism of chloroplast division are rings of polymerised molecules, that encompass the 
chloroplast at its midpoint or equator and constrict to pull and/or squeeze in the two chloroplast envelope membranes in a coordinated fashion. In the very latter stages of this constriction a fusion of the respective inner and outer envelope membranes takes place, to produce two entirely separated daughter chloroplasts.

In the process of chloroplast division, a ring of FtsZ proteins forms internal to the chloroplast inner envelope membrane on its stromal surface and composed of two FtsZ proteins, FtsZ1 and FtsZ2 (Vitha et al. 2001). These two proteins appear to form filaments spontaneously but produce a ring consisting of a fixed ratio of Ftsz1 and FtsZ2 proteins (Yoder et al. 2007; McAndrew et al. 2008). The FtsZ ring is tethered to the inner envelope membrane by the membrane spanning protein ARC6 (Pyke and Leech 1994; Vitha et al. 2003), which binds to the FtsZ2 protein specifically in the stroma and also has an envelope lumen domain.

External to the chloroplast on the cytoplasmic surface of the outer chloroplast envelope membrane, a punctated ring of ARC5/DRB5P protein forms, which overlays the internal FtsZ ring in a spatially coordinated manner. ARC5/ DRB5P is a dynamin-like protein (Gao et al. 2003; Miyagishima et al. 2003) and has been shown to exert constrictive force in vitro (Yoshida et al. 2006). ARC5/ DRB5P is recruited to the correct site on the chloroplast surface by two proteins PDV1 and PDV2 (Miyagishima et al. 2006). PDV1 forms a punctate ring on the envelope surface, in a similar way to ARC5/DRB5P, whereas PDV2 forms a continuous ring and appears to interact with ARC6 via the envelope luminal space, thereby linking the spatial integrity of the external ring to the internal ring (Glynn et al. 2008).

The makeup of the chloroplast division machinery is further complicated by the presence of two other rings, the plastid dividing rings (PD) (Kuroiwa et al. 1998), which have been described and analysed in most detail in algal chloroplasts (Miyagishima et al. 2001). An inner PD ring forms on the stromal surface of the inner envelope membrane and an outer PD ring forms on the cytoplasmic surface of the outer envelope membrane. In the red alga, Cyanidioschyzon merolae, these PD rings are composed of polyglucan filaments, associated with a glycogenin-like protein PDR1, which is probably involved in polyglucan synthesis (Yoshida et al. 2010).

These four rings together with associated proteins involved in their formation have been built into an ongoing model of the chloroplast division mechanism (Glynn et al. 2007; Maple and Moller 2010; Miyagishima et al. 2011). However, how the polyglucan plastid dividing rings interact with the FtsZ and dynamin rings remains unclear as do aspects of the process that control how this division machinery actually functions physically to constrict the plastid. Dynamin/FtsZ rings have been clearly shown to be able to apply constrictive force, as elegantly demonstrated in vitro experiments (Yoshida et al. 2006).

However what is very clear from many different experiments in which the expression of plastid division genes have been modified either by expression control or by mutagenesis, is that the chloroplast division system is highly flexible and compensatory in that perturbation of chloroplast division simply induces increased chloroplast expansion of the extant chloroplasts to give rise to a similar sized total chloroplast compartment in the cell (Pyke and Leech 1992, 1994; Osteryoung et al. 1998; Stokes et al. 2000).

Another important consideration is that when plastid division genes are manipulated using genetic and transgenic approaches, changes in chloroplast size and number are commonly observed in the resultant plants, but changes in the behaviour of proplastid division in the meristem are less obvious. Proplastids give rise to all of the plastids throughout the plant by virtue of their presence in the zygote and their continued division within the meristem regions of the plant. In fact, plastid division falls into two clearly distinct types: the division of proplastids in meristems to ensure proplastid continuity in cells leaving the meristem and differentiating, and a second phase of plastid division that happens with differentiated plastids during cell maturation in plant organs. This second phase is typified by chloroplast division in developing leaf mesophyll cells and has become the most researched mode of plastid division, although other differentiated plastids, such as amyloplasts are known to divide. However, in many cell types, the second phase of plastid division does not appear to take place. As a result in many cell types, including epidermal cells, root cells and vascular cells, the plastid population is relatively low and derived from those plastids provided in each cell by proplastid division in the meristem. This fact is often overlooked in plastid division biology and indeed it is an interesting question as to why the second phase of plastid division, as seen in chloroplasts, is absent in the vast majority of cells within the plant.

It has generally been assumed that different types of plastids such as proplastids, chloroplasts and amyloplasts use a similar mechanism by which to divide. Some evidence supports this, since manipulating FtsZ genes alters amyloplast division (de Pater et al. 2006). However, the assumption that proplastids divide using a chloroplast-type of division mechanism may well be flawed. Several lines of evidence suggest that proplastids may use some alternative type of mechanism by which to divide. Firstly, mutant phenotypes such as that of $\operatorname{arc} 6$, resulting into 2-3 giant chloroplasts in leaf mesophyll cells do not contain plastidless mesophyll cells (Pyke and Leech 1994) and thus it 
follows that in such mutant lines there must be some type of plastid division and segregation occurring in meristematic cells, otherwise the mutation would lead to large numbers of plastid-less cells and would most likely be lethal, which is obviously not the case. Secondly the ARC5/DR5BP dynamin-like division protein is not expressed in shoot apical meristem (Okazaki et al. 2009), suggesting that it is not functional in proplastid division. Thirdly, in plants completely lacking FtsZ proteins (Schmitz et al. 2009), plastid division can still occur suggesting some alternative mechanism for plastid division may well be functional in the background.

One possible explanation could be that different elements of the chloroplast division machinery operate in different ways in the division of different types of plastids. It could be that the plastid dividing rings and the FtsZ/ dynamin rings represent two different modes of plastid division, which can occur separately, or overlap, depending on the type of plastid or the stage of development. Hence the plastid dividing ring mode of division may be the major form of replication in proplastids in meristems whereas an FtsZ/dynamin based mode of division could be the main method of replication in chloroplasts. If this were the case, it would concur with the three lines of evidence mentioned earlier, in that proplastids use a different mode of plastid division to chloroplasts. A technical difficulty to date is that the plastid dividing rings have only been visualised in vivo by electron microscopy and no study has clearly shown an overlay of visualised FtsZ rings and plastid dividing rings in chloroplasts or proplastids from higher plants. One inherent problem with electron microscopy in this way is that the observation of electron dense rings around an isthmus or constriction on a dividing chloroplast could also be made of FTsZ/dynamin and the associated proteins. Is there a good reason why electron microscopy should differentiate between rings of proteins and rings of polyglucans? In addition, models of chloroplast division, which contain four concentric rings, seem overly complex for the division of a relatively simple organelle, and more complex than found in an analogous organelle the mitochondria, where the division machinery is apparently somewhat less complex (Logan 2010; Scott and Logan 2011).

Testing whether this suggestion is correct will require an in depth analysis of proplastid division in the meristems of higher plants. Unfortunately little is known about these somewhat enigmatic organelles, simply because of their small size and difficulty to access within small meristem tissues and cells. Electron micrographs of proplastids in meristem cells show them to be relatively consistent in shape with a central elongated isthmus for those in division (Oross and Possingham 1989; Robertson et al. 1995, 1996; Pyke 2009). However, the reality could be much different. In root meristem cells and in dividing cells in culture, the

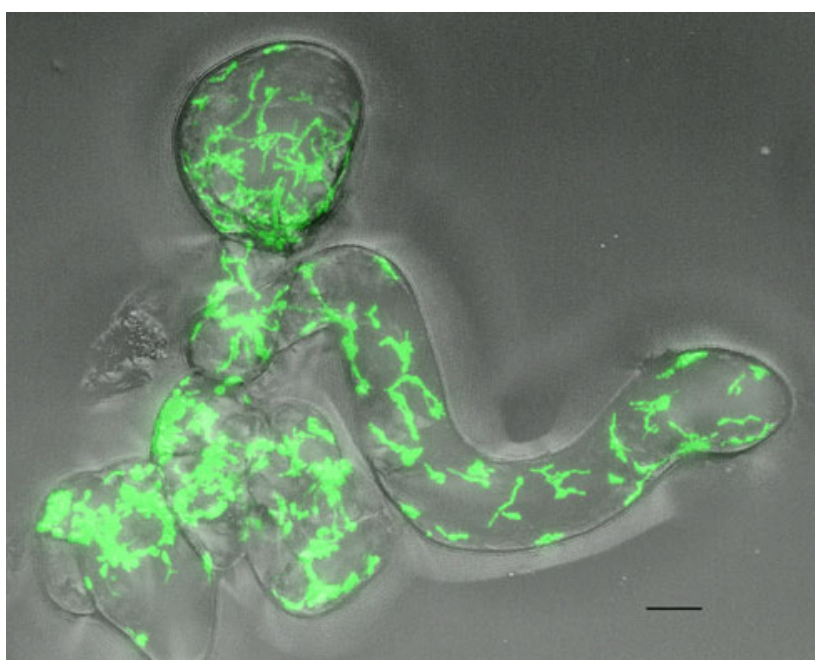

Fig. 1 A clump of cultured cells of tomato expressing plastidtargeted green fluorescent protein and revealing the highly heterogeneous morphology of proplastids within these dividing cells. Bar $=20 \mu \mathrm{m}$. Image by Linda Cholerton

visualisation of proplastids using fluorescent proteins shows them to be very heterogeneous indeed (Köhler et al. 1997, 2000; Pyke 2010; Fig. 1) with a wide array of plastid-derived structures including stromules and vesicles. Thus proplastid populations and their proplastid morphology are probably much more dynamic than those original electron microscope images might suggest. Consequently is it is difficult to envisage how a complex four ring division mechanism, as suggested in the model of chloroplast division, might efficiently divide such heterogeneous organelles in a regulated manner.

Another possible mechanism of plastid replication is that of budding off of vesicles from the plastid body, which has been shown to occur during chromoplast biogenesis from chloroplasts in ripening tomato fruit (Forth and Pyke 2006) but has yet to be clearly characterised in proplastid division in meristem cells.

The mode of plastid division in other differentiated types of plastids may also differ somewhat from that described for the chloroplast. In the division of amyloplasts in rice endosperm, the growth of a septum across the dividing amyloplasts has been clearly shown to be a part of amyloplast division, a process in which chloroplast division proteins such as FtsZ and PDV have a role (Yun and Kawagoe 2010). In addition, a budding type of mechanism also exists in amyloplast division in rice and wheat endosperm leading to a "beads on a string" type of arrangement of amyloplasts before they eventually separate, a process that appears to involve the ARC5/DR5BP protein (Langeveld et al. 2000; Yun and Kawagoe 2009). Therefore, a major challenge for the next phase of plastid division research is to elucidate exactly which mechanisms for plastid division occur in 
which plastid type in the plant and whether there is an overlap of mechanism or different subsets of proteins and structures for different types of plastids.

\section{Plastid morphology}

One clear observation that has been greatly enhanced by modern imaging technology is that plastids, especially nongreen plastids, come in a variety of shapes and sizes. Whereas the traditional view of the chloroplast is of an oblate spheroid type of shape, like a flattened rugby ball, there is no doubt that chloroplasts along with most other plastid types can change their shape extensively and in a highly dynamic way. During the process of division the constriction of the central dividing rings pull and squeeze in the two envelope membranes centrally leading to a central constriction. In some cases, as observed in the early days of plastid division research (Leech et al. 1981), this can lead to the formation of a longer isthmus. Exactly how an FtsZ/PD based division mechanism can give rise to a thin isthmus joining two small plastids is unclear, although it is clear however that chloroplast division in Arabidopsis does appear to form a relatively consistent structure at the chloroplast's midpoint (Vitha et al. 2001). The ability of the plastid and its envelope membranes to change shape is highlighted perfectly by its ability to form stromules, thin membranous tubules that extend from the plastid body and contain stroma but no thylakoids or chlorophyll (Hanson and Sattarzadeh 2011). Stromules occasionally interlink plastids together (Fig. 2), thus providing evidence for envelope fusion between two distinct plastids, as well as facilitating the movement of small molecules such as GFP (Köhler et al. 1997, 2000) and large complexes such as RUBISCO holoenzyme (Kwok and Hanson 2004), between two adjoined plastids. Such interlinking may only be transient if one considers the dynamic nature of stromules (Gunning 2005, 2009). Larger structures such as nucleoids and ribosomes do not appear to move between plastids through stromules of differentiated plant tissues (Newell et al. 2012). However, a recent study observing plastids expressing photoconvertible mEosFP (Schattat et al. 2012) suggests that fusion and molecule exchange may not occur despite the close proximity of stromules, thus disagreeing with earlier work.

Stromules themselves are enigmatic structures whose primary function is still somewhat unclear, although some experiments suggest that cellular stress might induce stromule formation through the hormone ABA (Gray et al. 2012). They are much more prevalent in non-green plastids which in general exist at lower densities in cells than do tightly packed chloroplasts in leaf mesophyll cells. Stromules should however be considered in a bigger context of plastid shape, since the irregular morphologies of



Fig. 2 A pericarp cell from a ripening tomato fruit of the mutant suffulta expressing plastid-targeted green fluorescent protein (Forth and Pyke 2006) reveals a wide range of plastid morphologies and stromules. Most of these structures lack chlorophyll and eventually become chromoplasts. Bar $=40 \mu \mathrm{m}$

plastids, such as those in root cells, makes it difficult to determine what is a stromule and what is the plastid body. In such cases, it might be more prudent to consider the plastid as an organelle with highly variable morphology, of which the chloroplast represents one of the more stable entities in terms of shape and in which stromules are simply the finest structure within the range of plastid morphologies. Observation of weird and wonderful shaped non-green plastids in root cells, ripening fruit cells or cultured cells (Pyke 2007, 2010; Figs. 1-3), elicits questions about plastid biology that have yet to be addressed by the research community. One such consideration is how is plastid shape maintained or are such plastids in a constant state of morphological flux? Certainly stromules have clearly been shown to be highly dynamic and move, extend and retract in a time scale of seconds (Gunning 2005, 2009); movement that is most likely powered externally by interaction with the actin microfilament part of the cell's cytoskeleton. If the remaining plastid body has a distinct morphology, there must be some mechanism, that maintains such shape. Whether such a mechanism is internal or external to the plastid is unclear. In theory an osmotically swollen organelle could maintain shape and this presumably is the situation in most "regularly" shaped chloroplasts. One interesting aspect of plastid biology which has been demonstrated recently is the existence of a stretch receptor system in the plastid envelope (Haswell and Meyerowitz 2006; Veley et al. 2012) similar to that found in the osmotic response system in bacteria. Such a system 


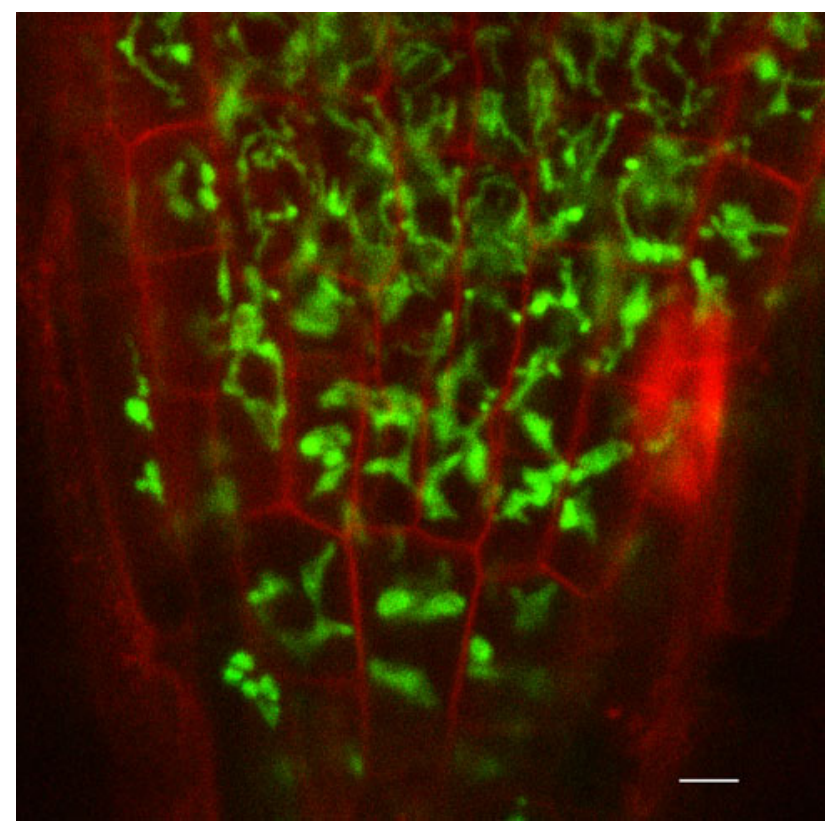

Fig. 3 Plastids in the root tip of Arabidopsis visualised with plastidtargeted green fluorescent protein showing the complexity of plastid morphologies in this tissue. Cell walls are fluorescently stained with propidium iodide. Bar $=20 \mu \mathrm{m}$. Image by Lukas Muller

could well provide the key to many aspects of plastid biology and explain how the plastid monitors its shape and morphology, but in non-green plastids, where morphologies are inherently variable and unlike that seen in chloroplasts in leaf mesophyll cells, the situation appears different. It is difficult to conceive how an osmotic system could enable shape stability in complex plastid shapes and indeed in large chloroplasts mutants, such as arc6, where isolated chloroplasts retain their shape in different osmotica (Pyke 2007). An internal structural support network could well stabilise plastid morphologies, although the evidence for such a structure is weak. The existence of an internal plastoskeleton has been reported (Reski 2002) but has not been generally proven, although it is conceivable that an FtsZ based internal skeleton could provide scaffolding for plastid morphology. Whilst stromules have been shown to be pulled in their extension by the cell's cytoskeleton of microfilaments and microtubules (Kwok and Hanson 2003), it is more difficult to conceive how this could function to provide a more long-term stable shape, although there is evidence for myosin actin binding within proteins in the outer plastid envelope (Sattarzadeh et al. 2009).

\section{Plastid budding and fusion}

One interesting concept that has been little considered is that of plastid fusion. Since stromules can join together plastids in a transient manner, it follows that the plastid envelope must be inherently capable of fusion with itself, yet such a process has not been described in plastids, in contrast to other organelles such as mitochondria in which organelle fusion is common place (Logan 2010; Scott and Logan 2011). Indeed the plastid envelope has to be able to fuse with itself since in the last stage of plastid division, central constriction enables the membrane to fuse and produce two separate organelles. Could it be that in some circumstances plastids can fuse, analogous to the process clearly shown in mitochondria? If stromules regularly get stretched and break, as has been shown (Gunning 2009), then the notion that the broken pieces might fuse back to the extant plastid population seems conceivable. As part of this concept, the idea that plastids can produce vesicles that arise by budding and can fuse with other plastids or organelles needs to be considered by the plastid community. There are several aspects of plastid biology that have become described in recently in which vesicle trafficking to and from plastids are required. Firstly budding chloroplasts in ripening tomatoes clearly show the production of vesicles derived from the plastid body, containing plastid-targeted GFP, and that probably differentiate into chromoplasts (Forth and Pyke 2006). Secondly the metabolism of fatty acids in plastids requires trafficking of molecules from the plastid to the endoplasmic reticulum and back again (Benning et al. 2006; Wang and Benning 2012), a process which has been well characterised at a metabolic level and more recently close interactions between the chloroplast and the ER have been shown, with distinct contact sites between the chloroplast envelope and the ER (Schattat et al. 2011a, b). Thirdly, recent experiments have shown that plastid genomes can move between cells in plants (Stegeman and Bock 2012; Thyssen et al. 2012). This is a remarkable discovery, and it is suggested that small plastid derived structures containing plastid genomes could move through cell-cell junctions. Other experiments in which GFP was micro-injected into individual chloroplasts, which then spread to surrounding cells (Knoblauch et al. 1999), suggests that such a mechanism could exist. Alternatively stromules could exist which join together plastids in neighbouring cells, although such a system has not been reported to date. So although the fusion of whole plastids together is probably unlikely to happen, the production and fusion of plastid-derived vesicles or pieces of stromule with plastids is a distinct possibility.

\section{Outlook}

The past 20 years have revolutionised our view of plastids as endosymbiotic organelles and has shown them to be much more dynamic and integrated into their host's cell biology than once thought. There is still a huge amount to 
discover about their dynamic processing and cell biology, not least as to what happens with non-green plastid populations in cells other than chloroplast-containing mesophyll cells. Such cell biology is largely unexplored although the tools and technologies now exist to gain some handle on how non-green plastids function in different cell types in plants.

\section{References}

Benning C, Xu C, Awai K (2006) Non-vesicular and vesicular lipid trafficking involving plastids. Curr Opinion Plant Biol 9:241247

de Pater S, Caspers M, Kottenhagen M, Meima H, ter Stege R, de Vetten N (2006) Manipulation of starch granule size distribution in potato tubers by modulation of plastid division. Plant Biotechnol J 4:123-134

Forth D, Pyke KA (2006) The suffulta mutation in tomato reveals a novel method of plastid replication during fruit ripening. J Exp Bot 57:1971-1979

Gao H, Kadirjan-Kalbach D, Froehlich JE, Osteryoung KW (2003) ARC5, a cytosolic dynamin-like protein from plants, is part of the chloroplast division machinery. Proc Natl Acad Sci USA 100:4328-4333

Glynn JM, Miyagishima S-Y, Yoder DW, Osteryoung KW, Vitha S (2007) Chloroplast division. Traffic 8:451-461

Glynn JM, Froehlich JE, Osteryoung KW (2008) Arabidopsis ARC6 co-ordinates the division machineries of the inner and outer chloroplast membranes through interaction with the PDV2 in the intermembrane space. Plant Cell 20:2460-2470

Gray JC, Hansen MR, Shaw DJ, Graham K, Dale R, Smallman P, Natesan SKA, Newell CA (2012) Plastid stromules are induced by stress treatments acting through abscisic acid. Plant J 69:387398

Gunning BES (2005) Plastid stromules: video microscopy of their outgrowth, retraction, tensioning, anchoring, branching, bridging and tip-shedding. Protoplasma 225:33-42

Gunning BES (2009) Plant cell biology on DVD. Springer, Berlin

Hanson MR, Sattarzadeh A (2011) Stromules: recent insights into a long neglected feature of plastid morphology and function. Plant Physiol 155:1486-1492

Haswell ES, Meyerowitz EM (2006) MscS-like proteins control plastid size and shape in Arabidopsis thaliana. Curr Biol 16:1-11

Knoblauch M, Hibberd JM, Gray JC, van Bel AJ (1999) A galinstan expansion femtosyringe for microinjection of eukaryotic organelles and prokaryotes. Nat Biotechnol 17:906-909

Köhler RH, Cao J, Zipfel WR, Webb WW, Hanson MR (1997) Exchange of protein molecules through connections between higher plant plastids. Science 276:2039-2042

Köhler RH, Schwille P, Webb WW, Hanson MR (2000) Active protein transport through plastid tubules: velocity quantified by fluorescence correlation spectroscopy. J Cell Sci 113:3921-3930

Kuroiwa T, Kuroiwa H, Sakai A, Takahashi H, Toda K, Itoh R (1998) The division apparatus of plastids and mitochondria. Int Rev Cytol 181:1-41

Kwok EY, Hanson MR (2003) Microfilaments and microtubules control the morphology and movement of non-green plastids and stromules in Nicotiana tabacum. Plant J 35:16-26

Kwok EY, Hanson MR (2004) GFP-labeled Rubisco and aspartate aminotransferase are present in plastid stromules and traffic between plastids. J Exp Bot 55:595-604
Langeveld SMJ, Van Wijk R, Stuurman N, Kijne JW, de Pater S (2000) B-type granule containing protrusions and interconnections between amyloplasts in developing wheat endosperm revealed by transmission electron microscopy and GFP expression. J Exp Bot 51:1357-1361

Leech RM, Thomson WW, Platt-Aloia KA (1981) Observations of the mechanism of chloroplast division in higher plants. New Phytol 87:1-9

Logan DC (2010) Mitochondrial fusion, division, and positioning in plants. Biochem Soc Trans 38:789-795

Maple J, Moller SG (2010) The complexity and evolution of the plastid-division machinery. Biochem Soc Trans 38:783-788

McAndrew RS, Olson BJ, Kadirjan-Kalbach DK, Chi-Ham CL, Vitha S, Froehlich JE, Osteryoung KW (2008) In vivo quantitative relationship between plastid division proteins FtsZ1 and FtsZ2 and identification of ARC6 and ARC3 in a native FtsZ complex. Biochem J 412:367-378

Miyagishima S-Y (2011) Mechanism of plastid division: from a bacterium to an organelle. Plant Physiol 155:1533-1544

Miyagishima S-Y, Kabeya Y (2010) Chloroplast division: squeezing the photosynthetic captive. Curr Opin Microbiol 13:738-746

Miyagishima S, Takahara M, Kuroiwa T (2001) Novel filaments $5 \mathrm{~nm}$ in diameter constitute the cytosolic ring of the plastid division apparatus. Plant Cell 13:707-721

Miyagishima SY, Nishida K, Mori T, Matsuzaki M, Higashiyama T, Kuroiwa H, Kuroiwa T (2003) A plant-specific dynamin-related protein forms a ring at the chloroplast division site. Plant Cell 15:655-665

Miyagishima S, Froehlich JE, Osteryoung KW (2006) PDV1 and PDV2 mediate recruitment of the dynamin-related protein ARC5 to the plastid division site. Plant Cell 18:2517-2530

Miyagishima S-Y, Nakanishi H, Kabeya Y (2011) Structure, regulation and evolution of the plastid division machinery. Int Rev Cell Mol Biol 291:115-153

Newell CA, Natesan SK, Sullivan JA, Jouhet J, Kavanagh TA, Gray JC (2012) Exclusion of plastid nucleoids and ribosomes from stromules in tobacco and Arabidopsis. Plant J 69:399-410

Okazaki K, Kabeya Y, Suzuki K, Moria T, Ichikawa T, Matsui M, Nakanishi H, Miyagishima S-Y (2009) The PLASTID DIVISION1 and 2 components of the chloroplast division machinery determine the rate of chloroplast division in land plant cell differentiation. Plant Cell 21:1769-1780

Oross JW, Possingham JV (1989) Ultrastructural features of the constricted region of the dividing plastids. Protoplasma 150:131138

Osteryoung KW, Vierling E (1995) Conserved cell and organelle division. Nature 376:473-474

Osteryoung KW, Stokes KD, Rutherford SM, Percival AL, Lee WY (1998) Chloroplast division in higher plants requires members of two functionally divergent FtsZ gene families. Plant Cell 10:1991-2004

Pyke KA (2007) Plastid development and differentiation. In: Bock R (ed) Topics in current genetics, vol 19., Cell and molecular biology of plastidsSpringer, Heidelberg, pp 1-28

Pyke KA (2009) Plastid biology, 1st edn. Cambridge University Press, Cambridge UK

Pyke KA (2010) Plastid division. AoB Plants. doi:10.1093/aobpla/plq016

Pyke KA, Leech RM (1992) Chloroplast division and expansion is radically altered by nuclear mutations in Arabidopsis thaliana. Plant Physiol 99:1005-1008

Pyke KA, Leech RM (1994) A genetic analysis of chloroplast division in Arabidopsis thaliana. Plant Physiol 104:201-207

Reski R (2002) Rings and networks: the amazing complexity of FtsZ in chloroplasts. Trends Plant Sci 7:103-105

Robertson EJ, Pyke KA, Leech RM (1995) arc6, a radical chloroplast division mutant of Arabidopsis also alters proplastid 
proliferation and morphology in shoot and root apices. J Cell Sci 108:2937-2944

Robertson EJ, Rutherford SM, Leech RM (1996) Characterisation of chloroplast division using the Arabidopsis mutant arc5. Plant Physiol 112:149-159

Sattarzadeh A, Krahmer J, Germain AD, Hanson MR (2009) A myosin XI tail domain homologous to the yeast myosin vacuolebinding domain interacts with plastids and stromules in Nicotiana benthamiana. Mol Plant 2:1351-1358

Schattat M, Barton K, Baudisch B, Klösgen RB, Mathur J (2011a) Plastid stromule branching coincides with contiguous endoplasmic reticulum dynamics. Plant Physiol 155:1667-1677

Schattat M, Barton K, Mathur J (2011b) Correlated behaviour implicates stromules in increasing the interactive surface between plastids and ER tubules. Plant Signal Behav 6:715-718

Schattat MH, Griffiths S, Mathur N, Barton K, Wozny MR, Dunn N, Greenwood JS, Mathur J (2012) Differential colouring reveals that plastids do not form networks for exchanging macromolecules. Plant Cell 24:1465-1477

Schmitz AJ, Glynn JM, Olson BJSC, Stokes KD, Osteryoung KW (2009) Arabidopsis FtsZ2-1 and FtsZ2-2 are functionally redundant, but FtsZ-based plastid division is not essential for chloroplast partitioning or plant growth and development. Mol Plant 2:1211-1222

Scott, I, Logan, DC (2011) Mitochondrial dynamics. In: Kempken F (ed.) Plant mitochondria. Adv Plant Biol 1:31-63

Stegeman S, Bock R (2012) Exchange of genetic material between cells in plant tissue grafts. Science 324:649-651

Stokes KD, McAndrew RS, Figueroa R, Vitha S, Osteryoung KW (2000) Chloroplast division and morphology are differentially affected by overexpression of FtsZ1 and FtsZ2 genes in Arabidopsis. Plant Physiol 124:1668-1677

Thyssen G, Svab Z, Maliga P (2012) Cell-to-cell movement of plastids in plants. Proc Natl Acad Sci USA 109:2439-2443
Veley KM, Marshburn S, Clure CE, Haswell ES (2012) Mechanosensitive channels protect plastids from hypoosmotic stress during normal plant growth. Curr Biol 22:408-413

Vitha S, McAndrew RS, Osteryoung KW (2001) FtsZ ring formation at the chloroplast division site in plants. J Cell Biol 153:111-119

Vitha S, Froehlich JE, Koksharova O, Pyke KA, van Erp H, Osteryoung KW (2003) ARC6 is a J-domain plastid division protein and an evolutionary descendant of the cyanobacterial cell division protein Ftn2. Plant Cell 15:1918-1933

Wang Z, Benning C (2012) Chloroplast lipid synthesis and lipid trafficking through ER-plastid membrane contact sites. Biochem Soc Trans 40:457-463

Yoder DW, Kadirjan-Kalbach D, Olson BJ, Miyagishima SY, Deblasio SL, Hangarter RP, Osteryoung KW (2007) Effects of mutations in Arabidopsis FtsZl on plastid division, FtsZ ring formation and positioning, and FtsZ filament morphology in vivo. Plant Cell Physiol 48:775-791

Yoshida Y, Kuroiwa H, Misumi O, Nishida K, Yagisawa F, Fujiwara T, Nanamiya H, Kawamura F, Kuroiwa T (2006) Isolated chloroplast division machinery can actively constrict after stretching. Science 313:1435-1438

Yoshida Y, Kuroiwa H, Misumi O, Yoshida M, Ohnuma M, Fujiwara T, Yagisawa F, Hirooka S, Imoto Y, Matsushita K, Kawano S, Kuroiwa T (2010) Chloroplasts divide by contraction of a bundle of nanofilaments consisting of polyglucan. Science 329:949-953

Yun M-S, Kawagoe Y (2009) Amyloplast division progresses simultaneously at multiple sites in the endosperm of rice. Plant Cell Physiol 50:1617-1626

Yun M-S, Kawagoe Y (2010) Septum formation in amyloplasts produces compound granules in the rice endosperm and is regulated by plastid division proteins. Plant Cell Physiol 51: 1469-1479 